\title{
Multiphase coupling of a reservoir simulator and computational fluid dynamics for accurate near-well flow
}

Hemmingsen, Casper Schytte; Glimberg, Stefan Lemvig; Quadrio, Nathan; Völcker, Carsten; Nielsen, Kenny Krogh; Walther, Jens Honore; Byrne, Michael; Engsig-Karup, Allan P.

Published in:

Journal of Petroleum Science and Engineering

Link to article, DOI:

10.1016/j.petrol.2019.03.063

Publication date:

2019

Document Version

Peer reviewed version

Link back to DTU Orbit

Citation (APA):

Hemmingsen, C. S., Glimberg, S. L., Quadrio, N., Völcker, C., Nielsen, K. K., Walther, J. H., Byrne, M., \& Engsig-Karup, A. P. (2019). Multiphase coupling of a reservoir simulator and computational fluid dynamics for accurate near-well flow. Journal of Petroleum Science and Engineering, 178, 517-527.

https://doi.org/10.1016/j.petrol.2019.03.063

\section{General rights}

Copyright and moral rights for the publications made accessible in the public portal are retained by the authors and/or other copyright owners and it is a condition of accessing publications that users recognise and abide by the legal requirements associated with these rights.

- Users may download and print one copy of any publication from the public portal for the purpose of private study or research.

- You may not further distribute the material or use it for any profit-making activity or commercial gain

- You may freely distribute the URL identifying the publication in the public portal 


\title{
Multiphase Coupling of a Reservoir Simulator and Computational Fluid Dynamics for Accurate Near-well Flow
}

\author{
Casper Schytte Hemmingsen ${ }^{\mathrm{a}, \mathrm{b}, \mathrm{c}}$, Stefan Lemvig Glimberg ${ }^{\mathrm{d}}$, Nathan \\ Quadrio $^{\mathrm{a}}$, Carsten Völcker ${ }^{\mathrm{a}}$, Kenny Krogh Nielsen ${ }^{\mathrm{d}}$, Jens Honore \\ Walther $^{b, c, e}$, Michael Byrne ${ }^{\mathrm{d}}$, Allan P. Engsig-Karup ${ }^{\mathrm{a}, \mathrm{c}}$ \\ ${ }^{a}$ Department of Applied Mathematics and Computer Science, Technical University of \\ Denmark, Building 324, DK-2800 Kgs. Lyngby, Denmark \\ ${ }^{b}$ Department of Mechanical Engineering, Technical University of Denmark, Building 404, \\ DK-2800 Kgs. Lyngby, Denmark \\ ${ }^{c}$ Center for Energy Resources Engineering (CERE), Technical University of Denmark, \\ DK-2800 Kgs. Lyngby, Denmark. \\ ${ }^{d}$ Lloyd's Register Consulting, Strandvejen 104A, DK-2900, Hellerup \\ ${ }^{e}$ Computational Science and Engineering Laboratory, ETH Zürich, Clausiusstrasse 33, \\ CH-8092 Zürich, Switzerland
}

\begin{abstract}
Near-well flow analysis is an important tool for gaining detailed insight of the flow behaviour and for improving well design and production optimization of real reservoirs. One challenge of accurate numerical modelling of the flow field in the vicinity of the well is related to the scale disparity factor in space and time. The numerical scale gap between the reservoir and the wellbore justifies the representation of a well as a point or line sink/source term in traditional reservoir models. However, standard numerical techniques for reservoir simulation are incapable of resolving the near-singular character of the pressure field in the vicinity of the well. Under the assumption that all length scales have impact on flow patterns, we present a proof-of-concept study aimed at improving the quality of the numerical simulation by considering the geometry and fluid flow near the wellbore in a fully connected system, thus accounting for the fine scale phenomena by means of a hybrid Navier-Stokes/Darcy wellbore model coupled with a full scale reservoir model. A weak coupling method based on fixed-point iterations, that preserves the mass flux transport across the coupled interface, while adjusting productivity indices, is demonstrated via numerical experiments. Several
\end{abstract}


different numerical experiments are performed to demonstrate the versatility and the improved well performance insight that the coupled method offers, including horizontal well inflow profile, influence of formation damage and optimal well configuration.

Keywords: Multiphase Flow, Porous Media, CFD, Near-wellbore, Reservoir Simulation

\section{Introduction}

Fluid dynamics in the vicinity of the wellbore has a considerable impact on well productivity. In the last decade, many ultra-deep, long horizontal, multilateral wells have been drilled. These wells can be very expensive and may lead to complex wellbore flows. Accurate modelling of the flow behaviour in the vicinity of the wellbores, including frictional losses, multiphase flow effects, gas hydrates, is crucial for effective field operations. Classical well models are based on several assumptions and cannot fully describe the kind of behaviour or effects present in modern smart wells with downhole equipment [1]. Therefore, more complicated well models need to be developed, in which conservation laws describing fluid dynamics in wells are discretised and solved $[2,3,4]$.

General reservoir simulators use simple well models to relate the injection or production with the sandface pressure within a computational grid block [5]. At best, the well is treated as a boundary condition coupled with a simplified one-dimensional wellbore model. Two types of well models have mostly been used in numerical simulators, namely conventional and advanced well models. Conventional well models treat the wellbore as a single unit and provide a detailed analysis of fluid flow in standard vertical and deviated wells [6]. Density variations along the well and frictional losses are accounted for approximately [7]. Whilst advanced well models can handle complex geometries, increased length of perforated intervals and presence of control devices downhole in smart wells $[8,9,10]$. However, despite the sophistication of these advanced well models, the predictions made with them in some cases do not match the actual performance. Therefore, a model of the wellbore that takes into account the geometry and the near-well flow physics would lead to a powerful tool to gain improved insight in the flow dynamics and in the predictability of the solution, especially the production

rates that determine the net present value of a reservoir. Including more 
physics via improving the modelling basis in a full-field reservoir model is not only computationally demanding, but also difficult to account for at all relevant reservoir scales.

Several approaches have been developed to tackle this problem, particularly popular are techniques of domain decomposition [11, 12], local-global near-well upscaling $[13,14,15]$, multiscale methods [16] and coupled simulations $[10,17,18,19,20,21,22]$. In the domain decomposition approach, the field is divided into non-overlapping subdomains classified as near-well zones or reservoir zones. Each subdomain is then solved separately using boundary conditions deduced from the neighbours. This procedure iterates until a certain threshold is reached or a convergence criteria is satisfied. Near-well upscaling with a local-global method uses a local fine scale simulation for the near-well region, with boundary conditions supplied from a global coarse scale simulation. Near-well fine scale parameters are upscaled to the coarse scale grid increasing accuracy of the coarse scale simulation. Multiscale methods have been presented as a robust alternative to upscaling the pressure equation in Darcy related flow problems and have proved to be efficient on large reservoir models $[23,24]$. In flow simulations, multiscale methods use the geological fine-scale models to solve the problem on a coarse model. This means for instance that rock properties are not upscaled onto the coarse level. Instead, fine-scale rock information is incorporated into the global solution through local subproblems. Although solutions of the flow equations are at the coarse level, the fine-scale velocity field can be reconstructed on the fine-scale as well. The underlying idea of the multiscale methods is thus to capture subgrid effects on coarse grids and to allow reconstruction of velocity or pressure fields on underlying fine grids [25]. In coupled simulations, two standalone simulators are often used, one for the full-field reservoir and one for the near wellbore region. The technique does not necessarily require code modifications and the data exchanges are performed through numerical productivity indices and boundary conditions. The advantages of this approach are several, but the main feature is its versatility. No significant code modifications are required, the near-well model can be coupled to any reservoir simulator, such that the full-field reservoir simulator can take into account near-well phenomena at any time when necessary.

In this paper, we present an extension of the latter approach, namely we adopt a model that - to our knowledge - has never been included in full-field reservoir simulators until now. In the near-well region, we propose a model that solves a hybrid formulation of the Navier-Stokes/Darcy equations. This 
technique is known in literature as Computational Fluid Dynamic (CFD) for well modelling $[26,27,28]$ and it is well suited for modelling the flow of multiple fluids through fractions of reservoirs, completions and wells. In recent years, there has been an increasing trend to use this powerful tool, mostly motivated by the inadequacy of the classical Peacemann model [1] to capture the detail and complexity of formation damage. Moreover, complex scenarios, such as unloading liquid drilling fluids, sand failure, the impact of changing wellbore shape and the complexity of certain well geometries can be included in the global assessment of the reservoir.

The main objective here is to frame a mathematical environment for the coupled modelling. We focus mainly on the scale disparity, i.e. the several order of magnitude difference between the characteristic length and time scale of each model, and the issues arising from the simultaneous simulation of near and far well flow behaviour. Boundary conditions for the near wellbore simulations are based on the full-field reservoir model, while the wellbore production rates are used to update the Productivity Index (PI) in the full-field model. The coupling technique is based on a fixed-point iteration scheme, which converges after a certain criteria is satisfied, making it possible to meet a user-defined tolerance while controlling accuracy of the

simulations. Through numerical experiments we first validate the coupling approach using a standard water injection benchmark problem, followed by a horizontal test case that account for some of the complexities of near-well flow characteristics.

\section{Multiphase Flow Models}

We first describe the full-field model based on Darcy's law and then we formulate the Navier-Stokes equations in a way that allows both free flow and flow through porous media under certain assumptions.

\subsection{Darcy Based Full-Field Reservoir Model}

The mass balance equations for an incompressible two-phase fluid (e.g. water and oil) flow in a porous medium can be stated as

$$
\frac{\partial\left(\phi S_{q}\right)}{\partial t}+\nabla \cdot \mathbf{u}_{q}=\mathrm{M}_{q}, \quad q=w, o
$$

where $S_{q}$ and $\mathbf{u}_{q}$ denote the saturation and the velocity of phase $q$, respectively, $\phi$ is the porosity of the medium, and M is a possible sink/source term. 
The transport of phases is driven by spatial differences in pressure, $p$, and gravity, g, which can be described by Darcy's law since it has low to moderate velocities (here in absence of capillary effects)

$$
\mathbf{u}_{q}=-\frac{\mathbf{K}_{q}}{\mu_{q}}\left(\nabla p-\rho_{q} \mathbf{g} \nabla z\right)
$$

where $\mathbf{K}_{q}$ is the product of the absolute permeability $\mathbf{k}$ and the relative permeability $k_{r q}, \mu_{q}$ is the phase viscosity, and $\rho_{q}$ is the phase density. In order to close the problem, we note that the phases jointly fill the void space of the porous media, so the saturation equation holds

$$
\sum S_{q}=1, \quad q=w, o
$$

The well model representation using source/sink terms in reservoir simulators, relates the well inflow rate $\mathrm{Q}$ and the steady state pressure drawdown:

$$
\mathrm{Q}_{q}=\mathrm{PI}_{q} p^{d}
$$

where PI is the productivity index and the pressure drawdown, $p^{d}$, is defined as

$$
p^{d}=p^{\text {cell }}-p^{w}
$$

i.e. the difference between the block pressure containing a well and the wellbore pressure. The productivity index is given by

$$
\mathrm{PI}_{q}=\mathrm{WI} \cdot \lambda_{q}
$$

where $\lambda_{q}$ is the phase mobility defined as $k_{r q} / \mu_{q}$ and the well index, WI, is a constant value which depends on the ratio between the equivalent radius $r_{e}$ and the well radius $r_{w}$, the weighted horizontal absolute permeability $\sqrt{k_{x} k_{y}}$, the height of the cell $h$, and a skin factor $S$, using the radial form of the Darcy equation

$$
\mathrm{WI}=\frac{2 \pi \sqrt{k_{x} k_{y}} h}{\ln \left(r_{e} / r_{w}\right)+S} .
$$

The equivalent radius $r_{e}$ represents the radial distance from the well to the block pressure. Peaceman derived the following equation for the equivalent radius $r_{e}$ based on the assumptions of a block-centered vertical well, single phase flow, homogeneous reservoir, uniform grid, uniform permeability, at 
least five grid blocks to nearst boundary, and at least ten grid blocks to other wells [1]

$$
r_{e}=0.140365 \frac{\sqrt{\sqrt{k_{y} / k_{x}} \Delta x^{2}+\sqrt{k_{x} / k_{y}} \Delta y^{2}}}{\frac{1}{2}\left(\sqrt[4]{k_{y} / k_{x}}+\sqrt[4]{k_{x} / k_{y}}\right)}
$$

Several other attempts to improve well modelling can be found in the literature [29], but eventually all these techniques rely on the knowledge of the analytical pressure solution in the vicinity of the well. Although it is possible to derive analytical solutions for idealised cases, in general other numerical procedures are needed to account for near-well heterogeneities and the specific type of well completion. The model we propose will produce a well flow rate based only on the approximation of the phenomena happening in the wellbore region and without relying on parameter tuning.

\subsection{Hybrid Navier-Stokes/Darcy-Forchheimer Wellbore Model}

In the region near the wellbore a hybrid Navier-Stokes/Darcy-Forchheimer formulation is used. This model allows the simulation of fluid flow through porous media by means of a source term, which is activated in the control volumes defined as porous zone. The method is known as the One-Domain Approach (ODA) [30,31], and it has been implemented in several commercial multi-purpose softwares. The Navier-Stokes continuity equation for an isothermal, compressible and immiscible two-phase fluid can be stated as [32]

$$
\frac{\partial}{\partial t}\left(\phi \alpha_{q} \rho_{q}\right)+\nabla \cdot\left(\phi \alpha_{q} \rho_{q} \mathbf{u}_{q}\right)=0
$$

where $\alpha_{q}$ is the volumetric fraction. The phase conservation of momentum is defined as

$$
\begin{array}{r}
\frac{\partial}{\partial t}\left(\phi \alpha_{q} \rho_{q} \mathbf{u}_{q}\right)+\nabla \cdot\left(\phi \alpha_{q} \rho_{q} \mathbf{u}_{q} \mathbf{u}_{q}\right)= \\
-\phi \alpha_{q} \nabla\left(p+p_{c}\right)+\nabla \cdot\left(\phi \overline{\bar{\tau}}_{q}\right)+\phi \alpha_{q} \rho_{q} \mathbf{g}+\phi \mathbf{F}-\alpha_{q} \phi \mathbf{J}
\end{array}
$$

where $p_{c}$ is the capillary pressure, $\overline{\bar{\tau}}_{q}$ is the phase shear stress, $\mathbf{J}$ represents the momentum resistance source/sink term in a porous medium defined as

$$
\mathbf{J}=\alpha_{q} \phi \frac{\mu_{q}}{K_{q}} \mathbf{u}_{q}+\beta \rho_{q} \alpha_{q}^{2} \phi^{2}\left|\mathbf{u}_{q}\right| \mathbf{u}_{q}
$$


where $\beta$ is the Forchheimer coefficient [33]. To solve the pressure-velocity coupling we use an extended version of the SIMPLE algorithm, the phase coupled SIMPLE (PC-SIMPLE), which include closure relations for the interfacial coupling of momentum and turbulence in a Eulerian-Eulerian multiphase multi-fluid framework. The coupling terms are solved implicitly. Further details of the implementation is provided in [32]. Both the full-field reservoir model and the wellbore model are based on finite volume spatial discretisations, so we briefly recall the methodology.

\subsection{Finite Volume Method}

The discretisations carried on for both models in this paper are based on the finite volumes method and the two-point flux approximation (TPFA) scheme, which are briefly recalled in this section.

By further assuming single phase flow, (1) reads

$$
\nabla \cdot \mathbf{u}=\mathrm{q}, \quad \text { on } \Omega .
$$

If we partition the domain $\Omega$ into smaller volumes $\left\{\bar{\Omega}_{i}\right\}$, a finite volume solution will satisfy

$$
\int_{\bar{\Omega}_{i}} \nabla \cdot \mathbf{u} d \Omega=\int_{\partial \bar{\Omega}_{i}} \mathbf{u} \cdot \mathbf{n} d \Gamma=-\int_{\bar{\Omega}_{i}} \mathrm{q} d \Omega
$$

for each volume $\bar{\Omega}_{i}$, where $\mathbf{n}$ is the unit normal vector of the volume interface $\partial \bar{\Omega}_{i}$ pointing outward. By approximating the pressure with a cell-wise constant function $\mathbf{p}=\left\{p_{i}\right\}$ we can estimate the flux across cell interfaces $\gamma_{i j}=\partial \bar{\Omega}_{i} \cap \partial \bar{\Omega}_{j}$ from a set of neighbouring cell pressures.

To formulate the standard TPFA finite volume scheme we introduce a flow potential $\varphi=p+\rho \mathbf{g} z$ and solve for an equation of the type

$$
-\nabla \cdot \lambda \nabla \varphi=f
$$

where $\lambda$ is $K / \mu$. The TPFA scheme uses an interpolation of two neighbour cells, $\varphi_{i}$ and $\varphi_{j}$, to approximate the flux and gathers all the terms that do not involve cell potentials into an interface transmissibility $T_{i, j}$, e.g. the mobility which needs to be defined at the interface. Thus, the TPFA seeks a cell-wise constant function $\varphi=\left\{\varphi_{i}\right\}$ that satisfies

$$
\sum_{j} T_{i j}\left(\varphi_{i}-\varphi_{j}\right)=\int_{\Omega_{i}} f d \Omega, \quad \forall \Omega_{i} \subset \Omega .
$$


By definition, the fluxes are continuous across the interfaces and as a result the finite volume method is conservative.

After defining the two models separately, we now look at the technique for the coupled simulation. The method is well suited for commercial reservoir simulators, because they can easily take into account dynamical changes of the numerical PI and provide grid properties like pressure or saturation.

\section{Weakly Coupled Modelling Framework}

In many petroleum reservoir applications, highly sophisticated software codes are available to solve problems of different nature. Therefore it is desirable to use them in a modular way for multi-physics simulations, and this is particularly true, if an application requires the addition of further physical phenomena (for the case of this study, an example could be accounting e.g., formation damage or corrosion). The modular approaches used to establish a coupling algorithm are called weak [34] in contrast to strong algorithms which have a monolithic approach. The former schemes are often based on fixed-point iterations while the latter schemes are a pure implementation of Newton's method for full systems. In the following, a weak coupling algorithm is applied to relevant examples and some of the software implementation features are discussed. Generally, in multi-physics applications, the problem that one wants to solve can be summarized by finding a solution to the system of non-linear equations

$$
\mathbf{R}\left(\mathbf{x}^{*}\right)=0
$$

where $\mathbf{R}$ is called the residual and $\mathbf{x}^{*}$ is the solution of the non-linear system. In the framework of coupling involving multiple applications, (16) can be partitioned to reflect the contributions to the overall coupled problem arising from individual applications, e.g. from two coupled application $A$ and $B$ :

$$
\mathbf{x}=\left[\begin{array}{l}
\mathbf{x}_{A} \\
\mathbf{x}_{B}
\end{array}\right]
$$

where $\mathbf{x}_{A}$ are the unknowns from the application $A$ and $\mathbf{x}_{B}$ are the unknowns for the application $B$. The corresponding residual equation for the new partitioned variable reads

$$
\left[\begin{array}{l}
\mathbf{R}_{A}\left(\mathbf{x}_{A}^{*}, \mathbf{x}_{B}^{*}\right) \\
\mathbf{R}_{B}\left(\mathbf{x}_{A}^{*}, \mathbf{x}_{B}^{*}\right)
\end{array}\right]=0 .
$$


These equations represent constraints that need to be met and which can come for example from matching boundary conditions, matching fluxes (to make them unique at interfaces), and so on. Moreover, there is no limitation on the number of constraints as long as the computational cost is affordable.

From a software standpoint, the simplest mean of coupling multiple application codes together to achieve a multi-physics capability is based on some form of fixed-point iteration also known as successive substitution. This methodology is the most general and permits great flexibility when it comes to computational performance. The interaction between the solvers, in fact, is minimal, so we can run the codes in separate machines or with different parallelisation settings, e.g. we select the most suitable number of cores per solver depending on their computational complexity. A fixed-point iteration scheme consists of solving each domain in a sequence for its own problem variables while holding all the other variables fixed. Updated values are then exchanged and a coupled residual is evaluated in order to get measure of convergence and establish whether or not to repeat the procedure. Algorithm 1 outlines the overall procedure.

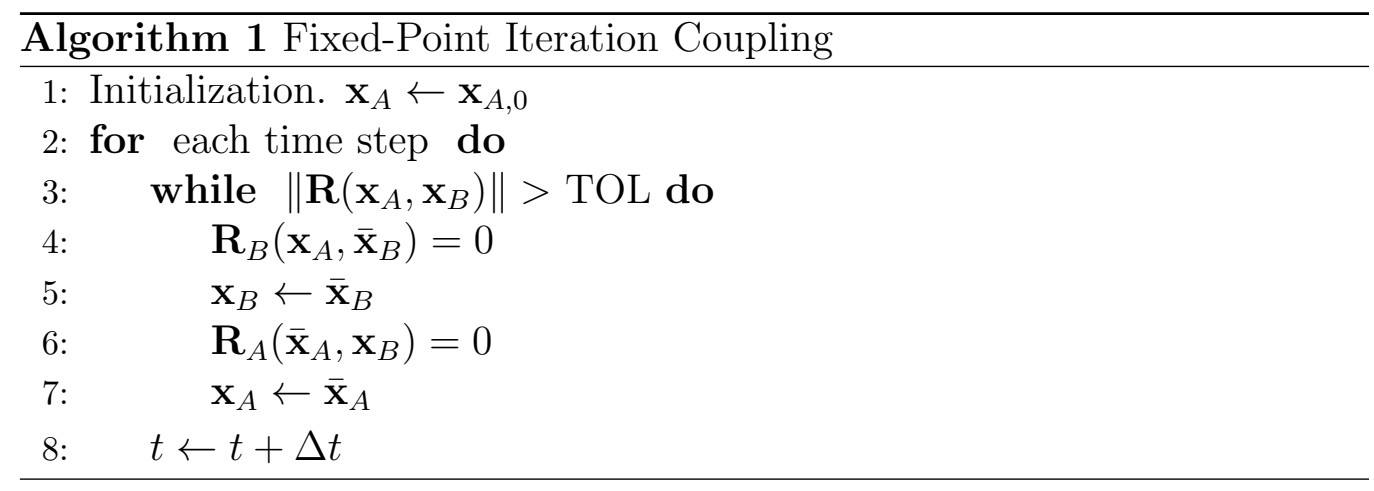

\subsection{Computational domain}

The weak coupling algorithm is designed for non-intrusive coupling of existing computational models. This implies that the algorithm is developed as an add-on to any prebuilt reservoir model, so no code modification of the full-field model is required. The near-well model represents a physically smaller region and its domain replaces the full-field simulator one in the vicinity of the well, see Fig. 1. The inlet to the near-well model shares the faces of the coarser resolution grid blocks of the reservoir model. Mass fluxes from the full-field model are then interpreted in the straightforward way on 


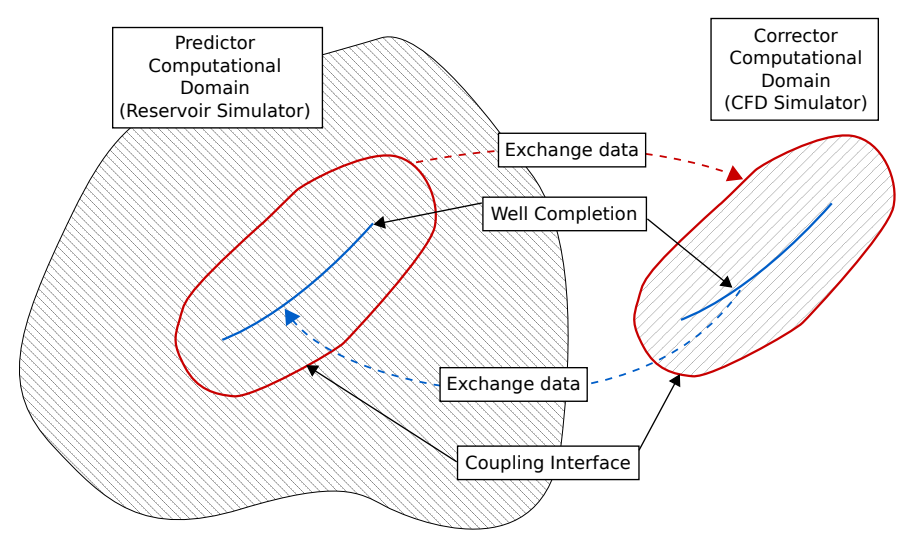

Figure 1: The illustration shows the concept of the data exchange between the two models.

the near-well model by weighting the fluxes with respect to differences in overlapping areas between cell faces, thus ensuring conservation of mass.

The source/sink terms used in the full-field model are updated using a dynamic upscaling approach. The numerical PIs of the full-field model is upscaled by

$$
\mathrm{PI}_{i, q}^{*}=\frac{\left\langle q^{w}\right\rangle_{i, q}}{\langle p\rangle_{i}-p^{b h p}}
$$

where the subscript $i$ indicates a variable from the near-well model evaluated within the coarse-grid reservoir well block, $\left\langle q^{w}\right\rangle_{i, q}$ is the summarized nearwell model flow rate of the individual phases evaluated at the sandface, $\langle p\rangle_{i}$ is the bulk-volume weighted average of the near-well pressure, and $p^{b h p}$ is the reference bottom hole pressure.

We replace the cell containing the well in the full-field simulator with an improved well model, obtained from the near-well model by interpolating or extrapolating the upscaled PI data.

$$
\mathrm{PI}_{i, q}^{t+\Delta t}=\mathrm{PI}_{i, q}^{*, T}\left(S_{\alpha}^{\text {cell }}, p^{\text {cell }}\right),
$$

where the superscript $T$ indicates a value evaluated at the coupling time step. The interpolation of the PI is substituted into equation 4. This quantity, coming from the CFD near well model, improves the fidelity of the flow simulation as it is computed with a more accurate model and less restrictive assumptions [1]. The increased accuracy is given by the different length scale of the phenomena that the model is able to simulate. However, the smaller 
the space scale, the smaller the time scale needs to be to have a stable numerical scheme. In the following section this concept is further illustrated.

\subsection{Time-scale separation}

The dynamical flow scales in the near-well region are much smaller in time and space compared to the reservoir flow. This results in large differences in time steps between the near-well model and the reservoir model. The performance of the fixed-point iteration will depend on the ability to handle this scale separation in the discretisation of both space and time. There are examples in literature, where the exploitation of time scale separation has a key role for the computational efficiency and the physical/numerical accuracy of the model [35]. In those applications, the simplest approach to time advancement is to set the time step of the model with the bigger scale (full-field reservoir) to be that required by the one with the smaller scale (wellbore). The exchange of information would be at every time-step, but of course no attempt is made to exploit scale separation and, as such, the computational cost will be prohibitive. Instead we use a variable coupling

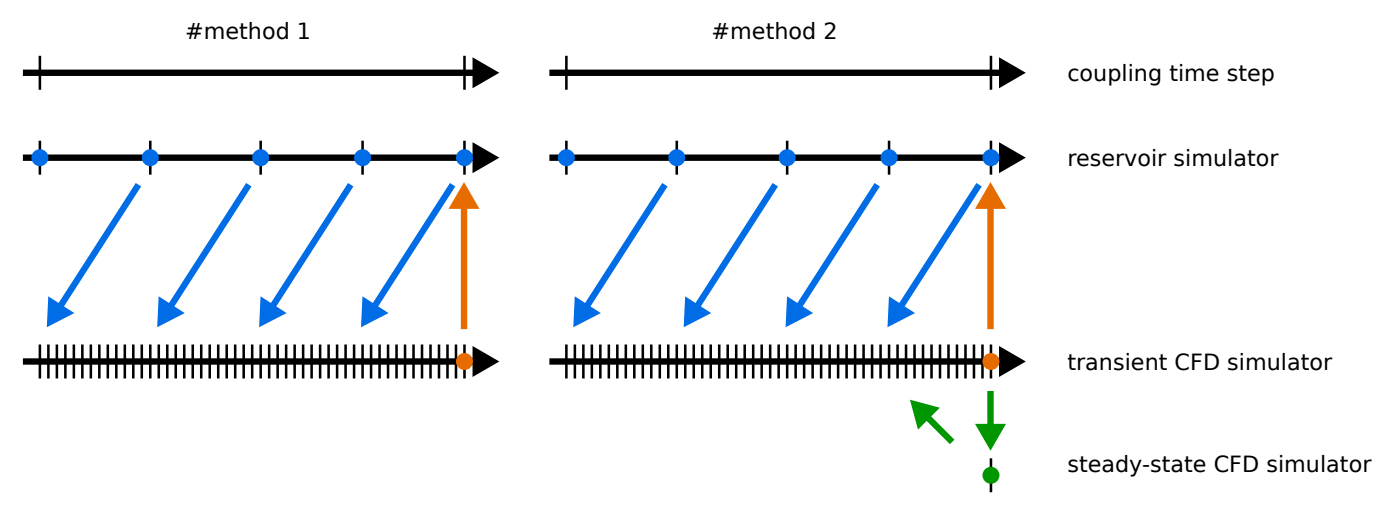

Figure 2: The scheme shows how the coupling is performed, i.e. for each full-field time step there is an iterative exchange of data. The blue color indicates transfer of data from the reservoir simulator to the transient CFD simulator, the green color indicates transfer of data between the transient CFD simulation and the steady state CFD simulation, and the orange color indicates the transfer of data at the end of the coupling time step.

time-step. Within each coupling time-step the full-field model is able to take several time-steps. The full-field results are then imposed on the near-well model at every full-field time-step, see Fig. 2. To avoid any discontinuities on the inlet of the near-well model, when imposing full-field results, the results are linearly interpolated in time. At the end of each coupling time-step 
upscaled PIs are sent to the full-field model, and if the two simulators agrees to within an acceptable tolerance, we move on to the next coupling step [21]. Furthermore, we test two different methods with different approaches to the scale separation. Method 1 uses the standard approach of the fixed-point iteration method, with a single model of the reservoir simulator and a single model for the near-well. If the time steps of the near-well model is much smaller than the reservoir model, the near-well model becomes the bottleneck of the coupling, e.g. when resolving fluctuations of vortex shedding, movement of a phase front in the well, etc.. Several near-well problems are, due to the computational cost, impractical using method 1. To remove the large time scale separation we introduce method 2. Method 2 uses a second fixedpoint iteration coupling within the near-well model. The near-well model is separated into two standalone near-well models, cf. Fig. 3, with the smallest scales collected in the same model. The smallest scales are typically within the vicinity of the well and within fractures. The near-well model with the larger time-steps are kept transient, as in method 1, and it contains the interface with the full-field model. Movement of any phase front are captured in the transient near-well model, until it reaches the second near-well model. The variation of the smallest time scales are nevertheless not imposed into

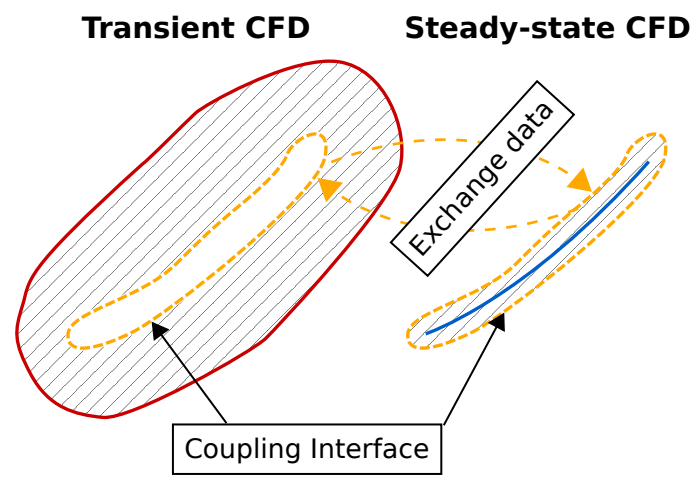

Figure 3: Extracted vicinity of the well in the near-well model used for coupling method 2.

the full-field model, due to the use of full-field time-steps, why we can assume a pseudo steady-state of the smallest scales in the second near-well model. The two models are coupled using the fixed-point iteration at the end of the coupling time step, cf. Fig. 2. The pseudo steady-state model receives data, that are constant in time, on the coupling interface. A solution is found when the model reaches a pseudo steady-state regime, and averaged pressure 
boundaries are sent back to the transient near-well model.

\subsection{Coupling intervals}

Variable coupling intervals are used to reduce the transfer of data between the two simulators. For large scale problems, with more than $10^{7}$ cells, it is time consuming to extract all the near-well data from the near-well model and calculate the upscaled PIs. The variable coupling intervals are controlled using three different monitors, evaluated at the end of the coupling time step. The first monitor reduces the coupling time-step if there is a moving phase front within the near-well model. The mass flow fraction on the near-well inlet and sandface are defined as

$$
M_{i}^{\mathrm{bc}}=\sum\left(\frac{\dot{m}_{i, s}^{\mathrm{bc}}+\dot{m}_{i, p}^{\mathrm{bc}}}{\sum\left(\dot{m}_{i, s}^{\mathrm{bc}}+\dot{m}_{i, p}^{\mathrm{bc}}\right)} \cdot \frac{\dot{m}_{i, s}^{\mathrm{bc}}}{\dot{m}_{i, s}^{\mathrm{bc}}+\dot{m}_{i, p}^{\mathrm{bc}}}\right)
$$

where $\dot{m}$ is the mass flow rate, the subscript $i$ indicates the face on the boundary, the subscript $s$ represent the secondary phase, and the subscript $p$ represent the primary phase. A difference above a certain tolerance indicates a moving front within the wellbore model.

$$
\left|M_{k}^{\text {inlet }}-M_{j}^{\text {outlet }}\right|>\epsilon
$$

The second monitor halves the coupling intervals until a certain tolerance for the mass flow rate on the inlet are respected.

$$
\left|\frac{\max \left(\dot{m}_{i, \alpha}^{\text {in }}\right)-\min \left(\dot{m}_{i, \alpha}^{\text {in }}\right)}{\max \left(\dot{m}_{i, \alpha}^{\text {in }}\right)}\right|>\epsilon .
$$

The third monitor ensures that the coupling interval is halved if the mass flow rate on the outlet is changing above a certain tolerance

$$
\left|\frac{\max \left(\dot{m}_{i, \alpha}^{\text {out }}\right)-\min \left(\dot{m}_{i, \alpha}^{\text {out }}\right)}{\max \left(\dot{m}_{i, \alpha}^{\text {out }}\right)}\right|>\epsilon .
$$

\section{Numerical Results}

The coupling algorithm is now investigated through several different cases of increasing complexity involving a vertical and a horizontal well. The CFD 
wellbore model is handled by Ansys Fluent, whereas the reservoir simulations are carried out in the open-source reservoir code MATLAB Reservoir Simulation Toolbox (MRST) [36]. As mentioned above, the flexibility of a fixed-point iteration scheme allows to choose freely the softwares and it does not require major code modifications. In the MRST-Fluent coupling a wrapper code is implemented to handle the co-simulation. The coupling algorithm is implemented in MATLAB. Furthermore, user-defined functions (UDFs) in ANSYS Fluent are used to interpolate the exchanged data on the boundaries of the CFD model. Fig. 4 shows a simple flow chart of the steps in the coupling algorithm.

\subsection{Case 1: 2D homogeneous waterflooding}

A simple piston-like displacement of oil by water is simulated. The model represents a two-phase, incompressible and immiscible flow with a vertical injector and producer. The reservoir is $220 \mathrm{~m} \times 20 \mathrm{~m} \times 20 \mathrm{~m}$, and we consider a cartesian grid with 11 blocks along the $x$-direction. The well experiences a non-uniform inflow due to the impermeable boundaries adjacent to the well block. This is a limitation of the Peaceman model when using a coarse grid. The wellbore model counts approximately $10^{3}$ cells, and the production well is modelled as a open-hole cylinder, see Fig. 5, imposing 2D effects to a 1D reservoir model. The rock and fluid properties of the reservoir model are listed in Table 1. The initial pressure is $11 \mathrm{MPa}$, we set a constant injection rate of $1.4 \mathrm{e}-4 \mathrm{~m}^{3} / \mathrm{s}$ and a constant bottom hole pressure of $10 \mathrm{MPa}$. The full-field model has a 1-day time-step and the simulation runs for 1,000 days. The wellbore model is able to run with time-steps of 0.1 day until a quasisteady state is reached. When the water front enters the wellbore model, the time-step is reduced to ensure stability of the oil-water interface on the fine grid. For the adaptive coupling time step we use a minimum coupling step of 5 days.

To test spatial convergence of the CFD simulations, we conduct a mesh sensitivity study using the near-well model from method 1. The study uses three different grids named mesh 1, mesh 2 and mesh 3 consisting of 759, 2653 and 7193 cells, respectively. For this mesh sensitivity study the near-well model is being simulated stand-alone without using the coupling framework. Water is injected at a constant injection rate of $1.0 \mathrm{e}-3 \mathrm{~m}^{3} / \mathrm{s}$ on the interface. The initial oil saturation is at the residual water saturation.

Fig. 6 shows the displacement of oil by water after 5 days and 10 days for all three meshes. The oil displacement is not well captured by mesh 1 , however 


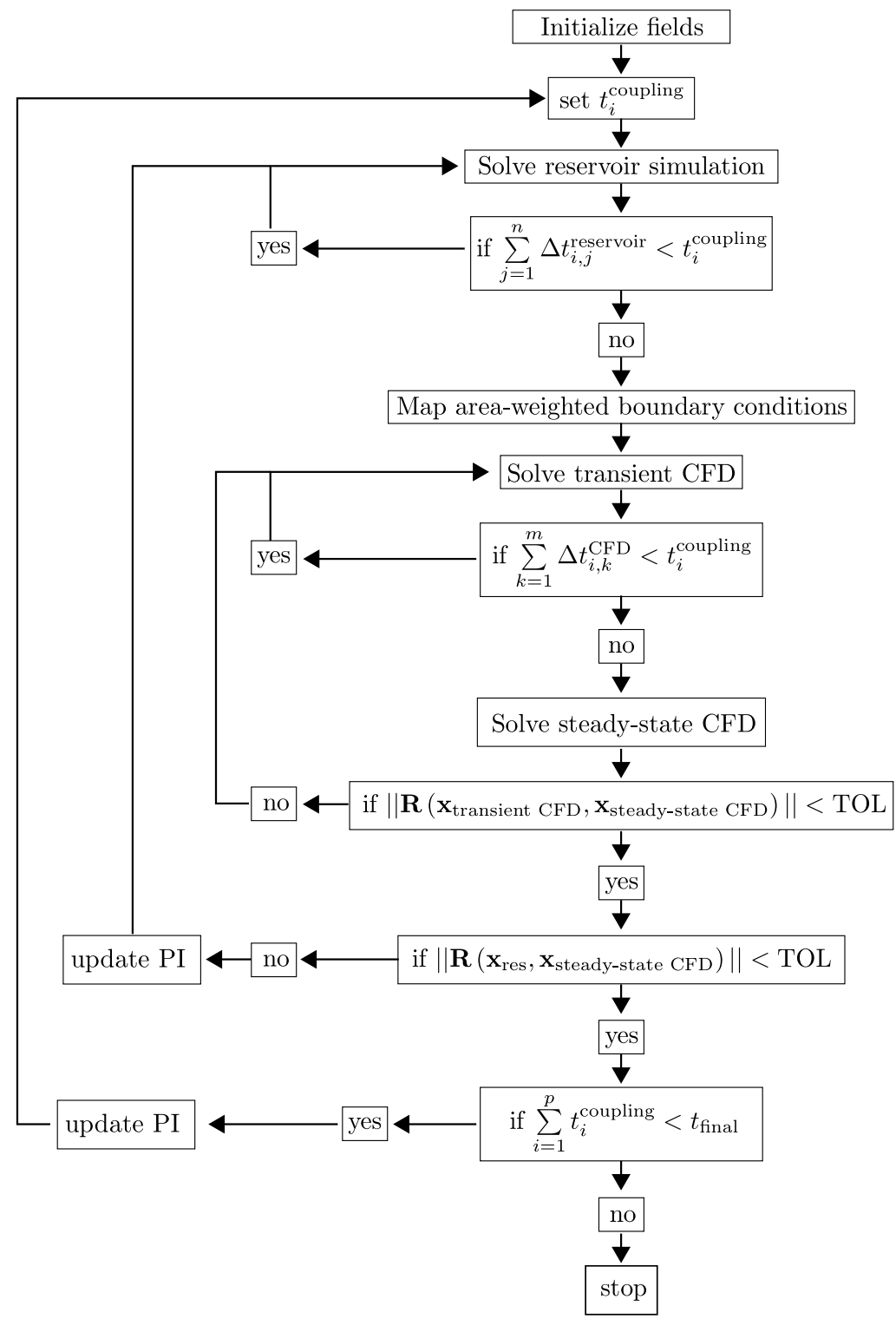

Figure 4: Flow chart showing the steps in the coupling algorithm. 


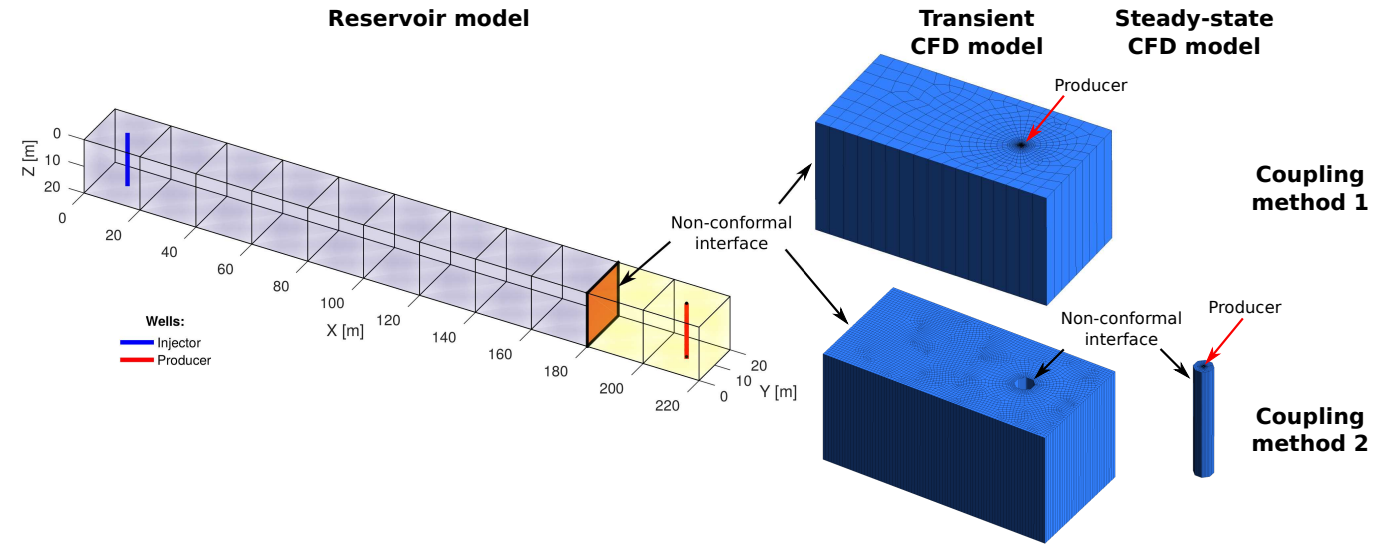

Figure 5: 1D reservoir model (left) coupled with a 3D wellbore model (right) for the producing well.

\begin{tabular}{|l|c|c|}
\hline Property & Value & Unit \\
\hline \hline Grid dimension & $11 \times 1 \times 1$ & - \\
\hline DX, DY, DZ & 20 & $\mathrm{~m}$ \\
\hline Porosity & 0.2 & - \\
\hline Permeability & 100 & $\mathrm{mD}$ \\
\hline Reference depth & 10 & $\mathrm{~m}$ \\
\hline Reference pressure & 110 & $\mathrm{bar}$ \\
\hline Rock compressibility & $45.4 \cdot 10^{-9}$ & $\mathrm{bar}^{-1}$ \\
\hline Water compressibility & $45.4 \cdot 10^{-9}$ & $\mathrm{bar}^{-1}$ \\
\hline Water viscosity & $0.3 \cdot 10^{-3}$ & $\mathrm{~Pa} \cdot \mathrm{s}$ \\
\hline Water density & 1000 & $\mathrm{~kg} / \mathrm{m}^{3}$ \\
\hline Oil density & 855 & $\mathrm{~kg} / \mathrm{m}^{3}$ \\
\hline Oil viscosity & $0.6 \cdot 10^{-3}$ & $\mathrm{~Pa} \cdot \mathrm{s}$ \\
\hline Oil formation volume factor* & 1 & $\mathrm{r} \mathrm{m} \mathrm{m}^{3} / \mathrm{st}{ }^{3}$ \\
\hline Well diameter & 0.215 & $\mathrm{~m}$ \\
\hline Bottom hole pressure & 100 & $\mathrm{bar}$ \\
\hline Injection rate & $1.4 \mathrm{e}-3$ & $\mathrm{~m}{ }^{3} / \mathrm{s}$ \\
\hline
\end{tabular}

\begin{tabular}{|c|c|c|c|}
\hline$s_{w}$ & $k_{r w}$ & $s_{O}$ & $k_{\text {row }}$ \\
\hline 0.1200 & $\overline{0}$ & 0.2000 & $\overline{0}$ \\
\hline 0.1723 & 0.0003 & 0.2523 & 0.0004 \\
\hline 0.2246 & 0.0025 & 0.3046 & 0.0029 \\
\hline 0.2769 & 0.0086 & 0.3569 & 0.0098 \\
\hline 0.3292 & 0.0204 & 0.4092 & 0.0233 \\
\hline 0.3815 & 0.0398 & 0.4615 & 0.0455 \\
\hline 0.4338 & 0.0688 & 0.5138 & 0.0787 \\
\hline 0.4862 & 0.1093 & 0.5662 & 0.1249 \\
\hline 0.5385 & 0.1631 & 0.6185 & 0.1864 \\
\hline 0.5908 & 0.2323 & 0.6708 & 0.2655 \\
\hline 0.6431 & 0.3186 & 0.7231 & 0.3641 \\
\hline 0.6954 & 0.4241 & 0.7754 & 0.4847 \\
\hline 0.7477 & 0.5506 & 0.8277 & 0.6292 \\
\hline 0.8000 & 0.7000 & 0.8800 & 0.8000 \\
\hline
\end{tabular}

Table 1: Rock and fluid properties for the 1D water flooding case. ${ }^{*}$ Oil is treated as incompressible dead oil and the formation volume factor is constantly equal to one irrespective of pressure. 


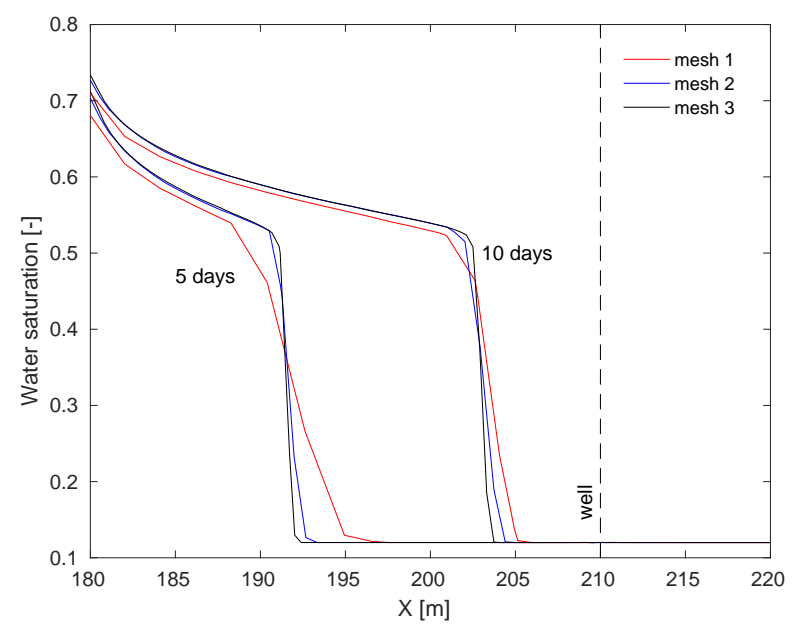

Figure 6: Saturation profile at $Y=10 \mathrm{~m}$.

as the mesh is refined the interface is well captured. In the remaining of this paper we uses cell sizes in the reservoir which corresponds to mesh 2 . Furthermore, time steps are kept sufficiently low to ensure stability and good convergence of the solver for a wide range of different boundary conditions.

We test the two different coupling methodologies mentioned above. The coupling method 1 is only feasible for cases with small differences in time scales across the domain. As the difference in time scale increases coupling method 2 becomes attractive.

As a reference case we use a reservoir model with local grid refinement (LGR) in the near-well region of the coarse 1D model. The reservoir model with LGR accounts for the non-uniform inflow to the well and consist of approximately $10^{4}$ cells. The LGR method is only valid for Darcy flow in porous media, why we only use it for validation for this simple case.

In Fig. 7 we present the production rates of both phases for the standard coarse grid, the coupled framework and the reference LGR method. We see a good match of water breakthrough between the coupled model and the LGR method, however, the standard coarse model inaccurately models the water breakthrough. The minor deviation between the reference case and the coupled method are due to the simple interpolation schemes and tolerances. 

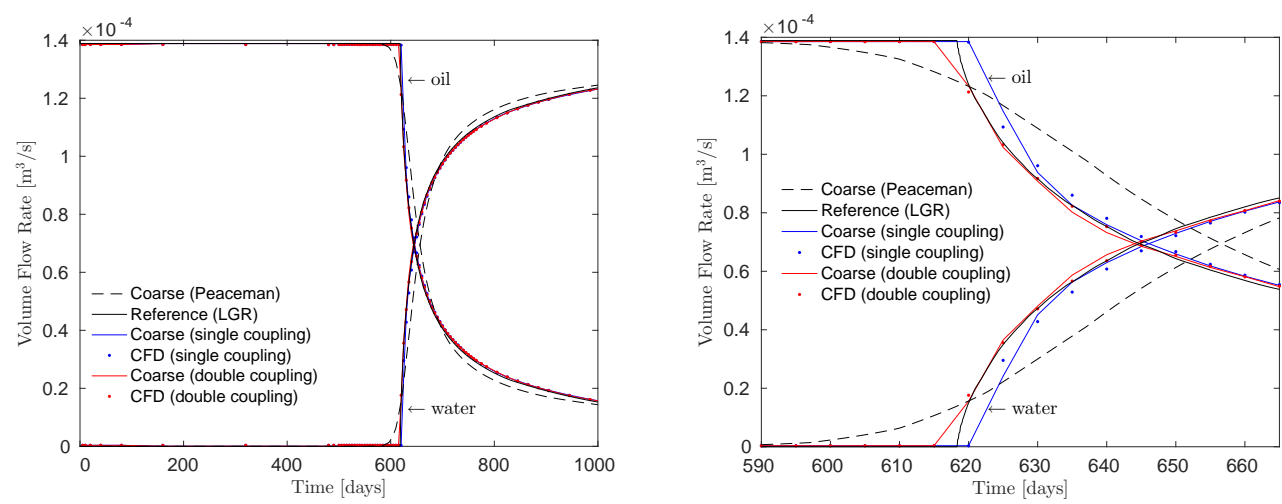

Figure 7: Production rates for water and oil. Left) the entire production period and right) a closeup of the water breakthrough.

Fig. 8 shows the pressure difference between the coarse well cell and the bottom hole. The coarse well cell pressure for the LGR method and the wellbore model is the volume average of the fine scale pressure. Both coupling methods shows a good agreement with the reference case. The Peaceman well model does not capture the correct level of the pressure for this simple case, even though the majority of the assumptions are met.

Pressure distribution in the wellbore model for the two different coupling methods are shown in Fig. 9. It should be noted that the asymmetric pressure around the well is captured with method 2, due to the additional fixed-point iteration coupling. Furthermore, in Fig. 10 the isolines of oil saturation are shown for the wellbore model at 6 different times. After water breakthrough some oil are trapped at the end of the domain.

\subsection{Case 2: Horizontal well with completion}

As a second case the inflow characteristics of a multi-segmented horizontal well are analyzed. As stated in the introduction, it is a challenge for reservoir well models to accurately account for pressure forces acting inside the well, formation damage effects, perforations, or the effect of an open annulus. This challenge is particularly stressed for horizontal wells, since flow rates are approximated by the Peaceman well model. Additional approximation techniques, such as flow performance tables, are introduced to leverage these issues. Even though such techniques are often comprehensive because they rely on well log and empirical data, the hybrid formulation on the other hand provides a good alternative. Since the geometry and the rock properties are 


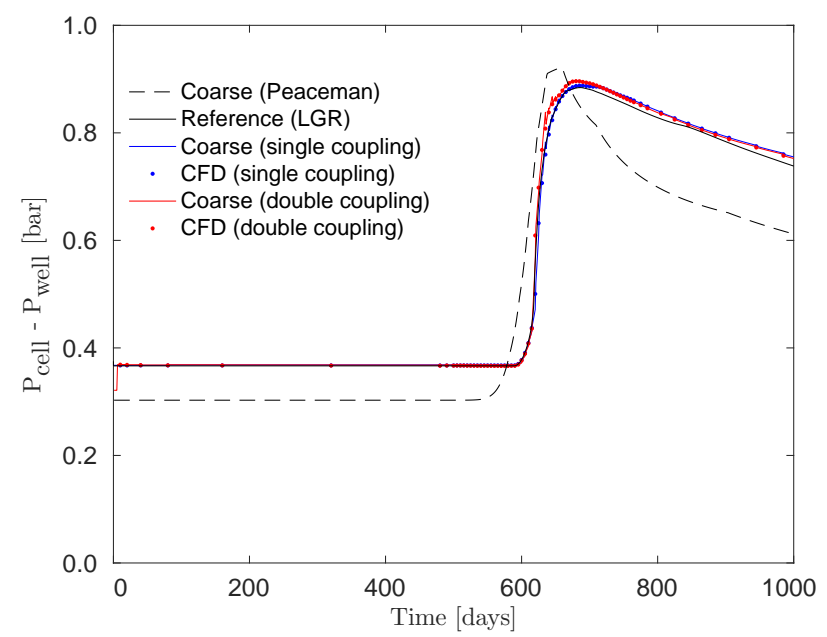

Figure 8: Pressure difference between well cell and bottom hole.
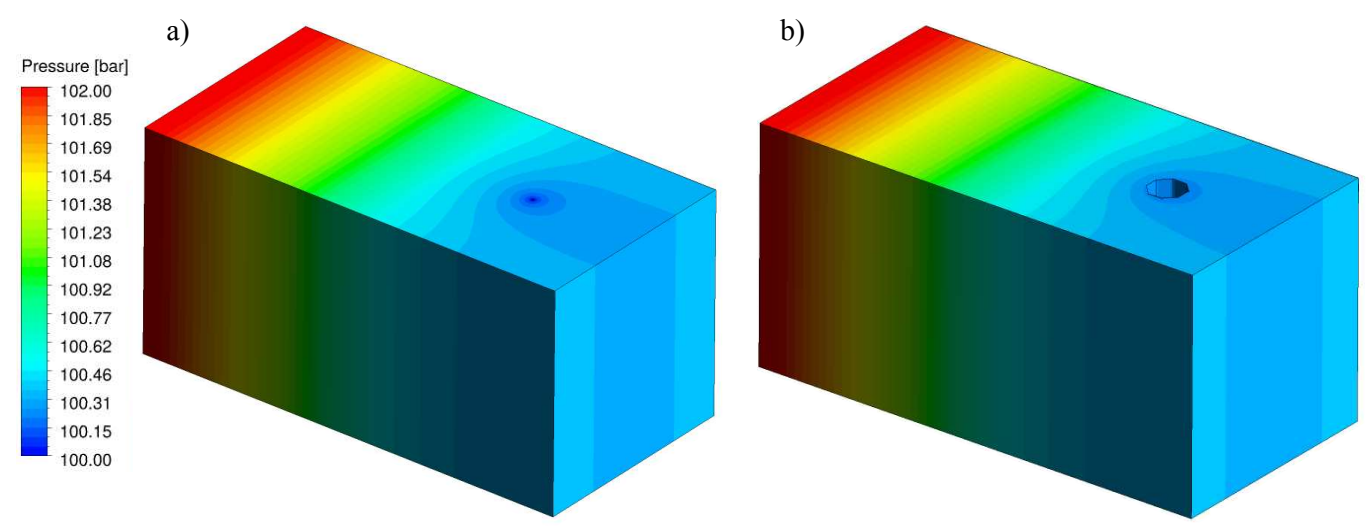

Figure 9: Pressure distribution in the wellbore model at 605 days for a) method 1 and b) method 2 . 


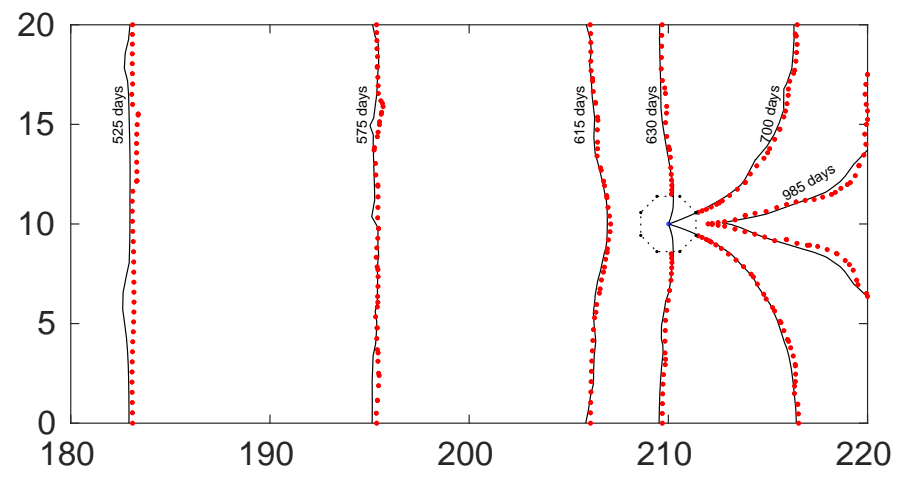

Figure 10: Isolines of oil saturation at 0.6 in the wellbore model at different days using method $1(-)$ and method $2(\bullet)$.

Zone 1

Zone 2

Zone 3

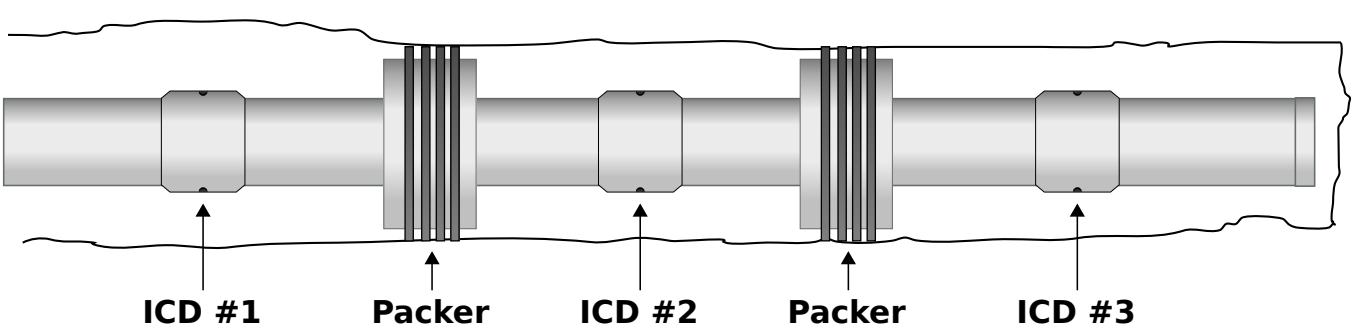

Figure 11: Sketch of well completion.

modelled as a fully connected system, in fact, the pressure drop and the flow information alongside the entire well is readily available, regardless of the well orientation and features.

The completion of the investigated horizontal multi-segmented well consists of three different zones, cf. Fig. 11. The well is segmented by two packers, each 2 meters long, dividing the well into three zones. Each zone is equipped with an inflow control device (ICD). Three different ICDs are used, cf. Table 2, which makes different characteristics of the individual ICD [37]. Information about the completion is defined in Table 2.

\begin{tabular}{|c|c|c|c|}
\hline Zone & ICD & Nozzle radius & nr. of nozzles \\
\hline \hline 1 & $\# 1$ & $5 \mathrm{~mm}$ & 2 \\
\hline 2 & $\# 2$ & $4 \mathrm{~mm}$ & 2 \\
\hline 3 & $\# 3$ & $3 \mathrm{~mm}$ & 2 \\
\hline
\end{tabular}

\begin{tabular}{|l|c|c|}
\hline Property & Value & Unit \\
\hline \hline Wellbore diameter & 0.15 & $\mathrm{~m}$ \\
\hline Inner diameter of casing & 0.10 & $\mathrm{~m}$ \\
\hline Outer diameter of casing & 0.12 & $\mathrm{~m}$ \\
\hline
\end{tabular}

Table 2: Details of (left) the inflow control devices and (right) the well. 


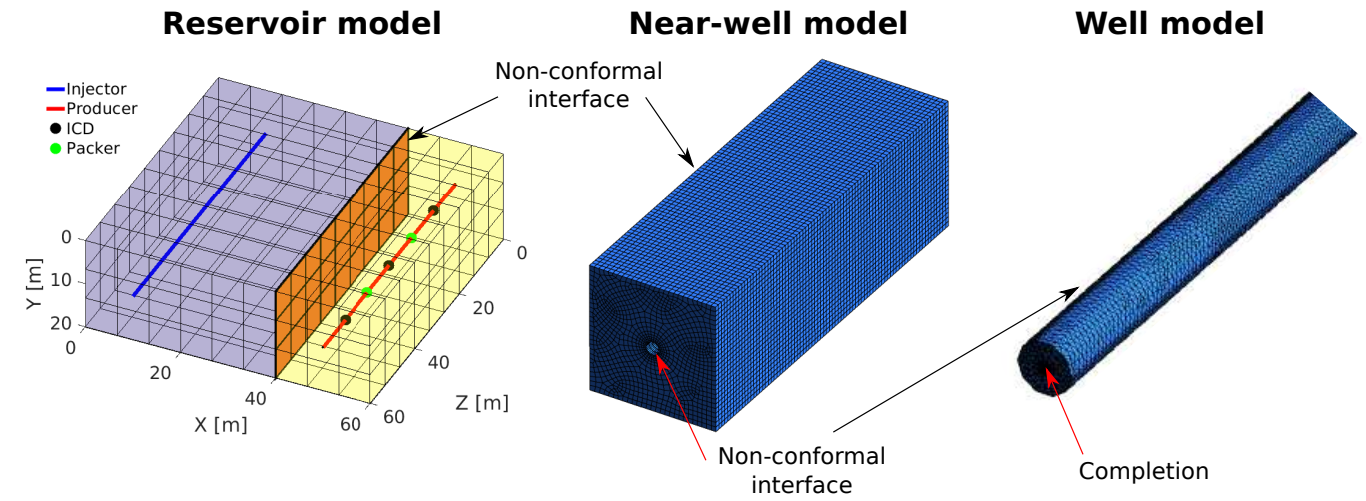

Figure 12: Spatial discretizations of the reservoir model, near-well model and the well model.

The horizontal well is located in a $60 \mathrm{~m} \times 20 \mathrm{~m} \times 60 \mathrm{~m}$ reservoir with gravity acting in the $y$-direction, cf. Fig. 12. An horizontal injector is horizontally located in the opposite side of the reservoir, but at the same vertical position. Fluid and rock properties are the same as in Case 1, except that the permeability in the horizontal direction is increased to $1000 \mathrm{mD}$ and the total water injection rate is set constant to $8.7 \mathrm{e}-4 \mathrm{~m}^{3} / \mathrm{s}$. The reservoir is initially saturated with oil. For the near-well model we include the closest grid cells, which results in a domain size of $20 \mathrm{~m} \times 20 \mathrm{~m} \times 60 \mathrm{~m}$. The pseudo steadystate model include the entire well and a cylindrical part of the reservoir that enclose the well. The cylindrical part has a radius of $1 \mathrm{~m}(3.28 \mathrm{ft})$ from the well center.

Fig. 13 shows that the highest production with the coupled multi-segment completion are from zone 1 . This is directly related to the flow characteristic of the ICDs, which is seen in the difference in production of each zone. The first water breakthrough happens in zone 1, a few days later in zone 2, and last in zone 3. It should be stressed that using the Peaceman model, each zone produces the same. Furthermore, the average pressure of the well cells in each zone is shown in Fig.14. Zone 1 has the lowest average pressure of the well cells, as expected.

Fig. 15 shows the pressure distribution in the transient near-well model. The influence of the different ICDs are easily observed, along with the influence of the packers on the pressure. Furthermore, we show the jet inside the well created by the the three ICDs, cf. Fig. 16. The highest velocities are seen in ICD \#3. 


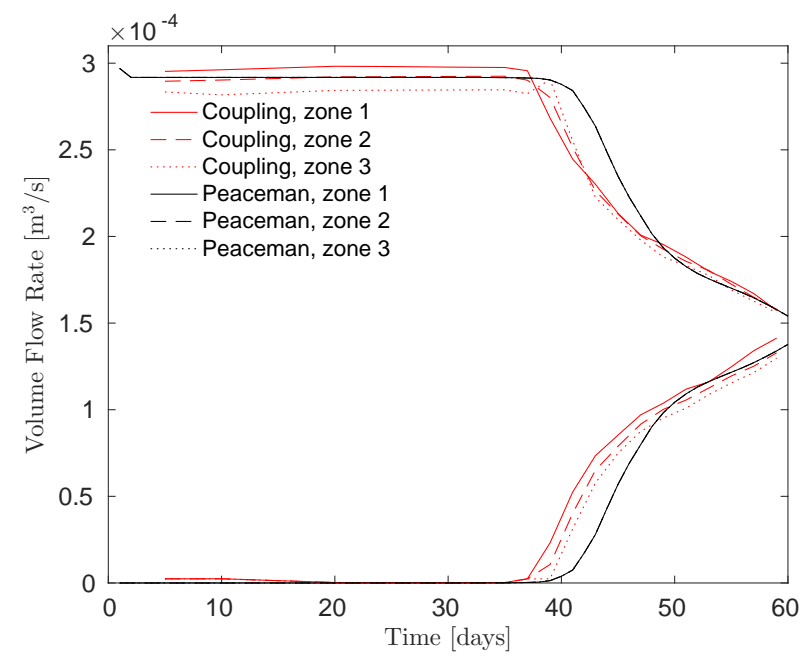

Figure 13: Production rates in the three different sectional zones.

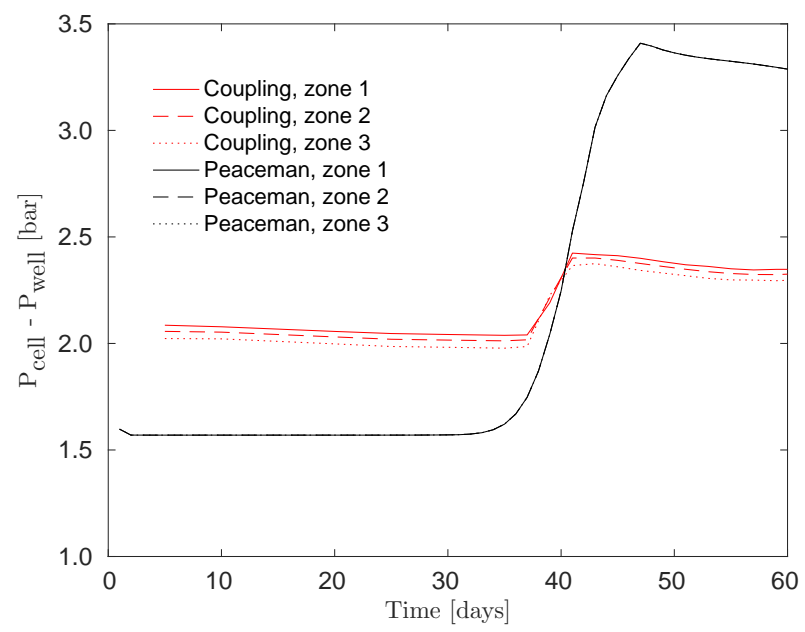

Figure 14: Difference between coarse well cell pressure and bottom hole pressure in the three different sectional zones. 


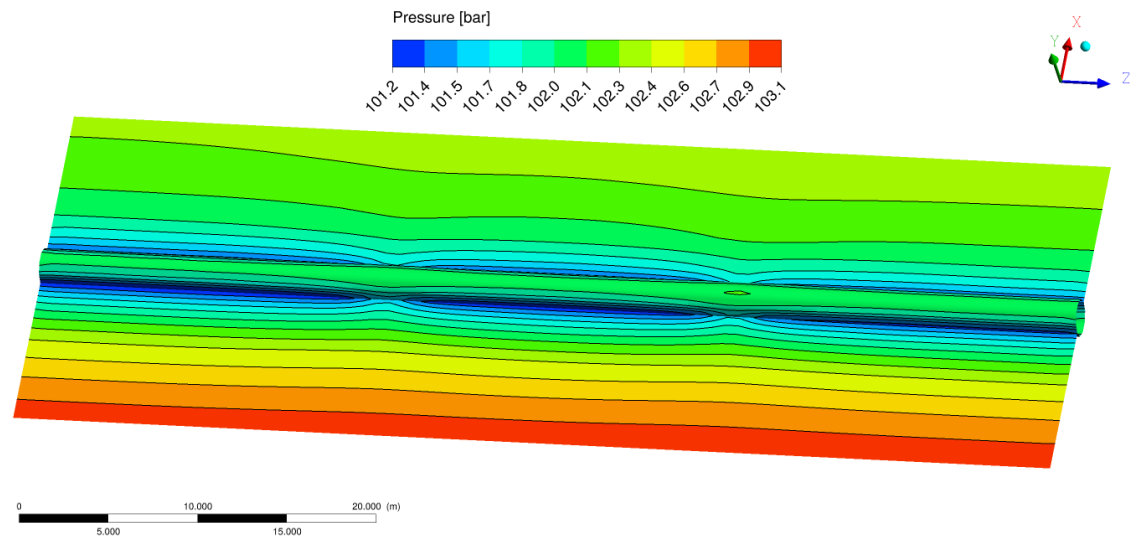

Figure 15: Plane cut at $y=10 \mathrm{~m}$ showing the pressure contours. Zone 1 is located at the left and the inlet is located at the bottom of the figure.
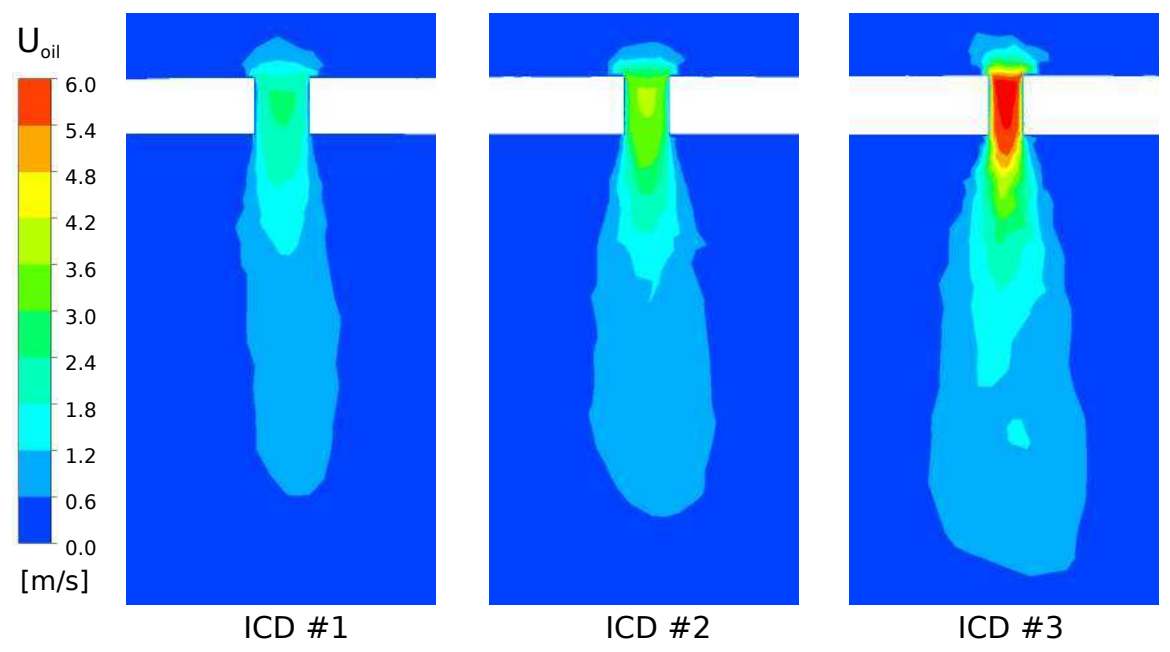

Figure 16: Oil velocity across the three different ICDs. 


\section{Conclusions and Future Work}

Providing a framework for multiphase coupling of a reservoir and a well model significantly increases the simulation details of the well performance and characteristics. Using a fixed-point iterative approach we are able to couple two different numerical methods and provide the best of the two worlds. Through two proof-of-concepts different scenarios were considered, where more information about well performance were obtained with the coupled models. The investigated cases has a low complexity, however the cases illustrate the objective of the present paper, to couple a reservoir simulator with computational fluid dynamic.

Using the coupling framework, it is possible to investigate the influence of complex near-well phenomena on the large and small length scales, e.g. fractured reservoirs, water and gas coning, formation damage, etc. The same level of details are not possible with any other current methods.

Even though the present work shows a good potential and new opportunities for reservoir modelling with improved near-well modelling, this new coupling framework does have limitations. The main limitations are the computational cost of running the detailed near-well modelling. At the moment the computational cost of detailed CFD makes it unattractive to run a real case for the entire lifetime of a reservoir. In ongoing work, we will investigate the use of model order reduction techniques to address this limitation.

\section{Acknowledgment}

The research was hosted by Department of Applied Mathematics and Computer Science (DTU Compute) and in close collaboration with Lloyds Register. The work has been supported by Innovation Fund Denmark (Grant No. 5184-00025B), Welltec A/S, Lloyds Register, Center for Energy Re-

sources Engineering (CERE), INEOS Oil \& Gas Denmark, and the Danish Center for Scientific Computing at the Department of Physics, DTU. We gratefully acknowledge the support on ANSYS Fluent software issues provided by ANSYS.

\section{References}

[1] D. Peaceman, Interpretation of well-block pressures in numerical reservoir simulation, SPE J. 18 (03) (1978) 183-194. 
[2] B. J. Dikken, Pressure drop in horizontal wells and its effect on production performance, J. Petroleum Technol. 42 (11) (1990) 1426-1433.

[3] L. Ouyang, Single Phase and Multiphase Fluid Flow in Horizontal Wells, PhD Thesis, Stanford University.

[4] Y. Jiang, Techniques for modeling complex reservoirs and advanced wells, PhD Thesis, Stanford University.

[5] S. Williamson, J. Chappelear, Representing wells in numerical reservoir simulation: Part 1 - theory, SPE J. 21 (1981) 323-338.

[6] E. Ozkan, Analysis of horizontal-well responses: Contemporary vs. conventional, SPE Reser. Eval. Eng. 4 (04) (2001) 260-269.

[7] GeoQuest, Eclipse technical description, multi-segment wells, Schlumberger.

[8] J. A. Holmes, T. Barkve, O. Lund, Application of a multisegment well model to simulate flow in advanced wells, Proc. European Pet. Conference 2 (1998) 171-181.

[9] J. A. Holmes, Modeling advanced wells in reservoir simulation, J. Petroleum Technol. 53 (11) (2001) 54-66.

[10] J. R. Augustine, An investigation of the economic benefit of inflow control devices on horizontal well completions using a reservoir-wellbore coupled model, Proc. European Pet. Conference (2002) 139-148.

[11] R. Ewing, R. Lazarov, Approximation of parabolic problems on grids locally refined in time and space, Applied Numerical Mathematics 14 (13) (1994) 199-211.

[12] S. Gaiffe, Hybrides et decomposition de domain pour la modelisation des reservoir petroliers, These de Doctorat, Universite Paris 6.

[13] T. Nakashima, L. Durlofsky, Accurate representation of near-well effects in coarse-scale models of primary oil production, Trans. Porous Media 83 (2010) 741-770.

[14] T. Nakashima, H. Li, L. Durlofsky, Near-well upscaling for three-phase flows, Comp. Geosci. 16 (1) (2012) 55-73. 
[15] H. Li, Y. Chen, D. Rojas, M. Kumar, Development and application of near-well multiphase upscaling for forecasting of heavy oil primary production, J. Pet. Sci. Eng. 113 (2014) 81-96.

[16] J. E. Aarnes, S. Krogstad, K.-A. Lie, Multiscale mixed/mimetic methods on corner-point grids, Comp. Geosciences 12 (3) (2008) 297-315.

[17] Y. Chen, L. Durlofsky, Adaptive local-global upscaling for general flow scenarios in heterogeneous formations, Trans. Porous Media 62 (2) (2006) 157-185.

[18] Y. Chen, Y. Li, Local-global two-phase upscaling of flow and transport in heterogeneous formations, Multiscale Modeling and Simulation 8 (1) (2009) 125-153.

[19] D. Y. Ding, Modeling formation damage for flow simulations at reservoir scale, SPE J. 15 (3) (2010) 737-750.

[20] S. Livescu, L. Durlofsky, K. Aziz, J. Ginestra, A fully-coupled thermal multiphase wellbore flow model for use in reservoir simulation, Journal of Petroleum Science and Engineering 71 (3-4) (2010) 138-146.

[21] D. Y. Ding, Coupled simulation of near-wellbore and reservoir models, J. Pet. Science Eng. 76 (1-2) (2011) 21-36.

[22] M. Karimi-Fard, L. J. Durlofsky, An expanded well model for accurate simulation of well-reservoir interactions, SPE Reservoir Simulation Symposium 20111 (2011) 688-699.

[23] P. Jenny, S. Lee, H. Tchelepi, Adaptive multiscale finite-volume method for multiphase flow and transport in porous media, Multiscale Modeling \& Simulation 3 (1) (2005) 50-64.

[24] H. Tchelepi, P. Jenny, S. Lee, C. Wolfsteiner, Adaptive multiscale finitevolume framework for reservoir simulation, SPE J. 12 (2007) 188-195.

[25] V. L. Hauge, Multiscale methods and flow-based gridding for flow and transport in porous media, Ph.d. thesis, Norwegian University of Science and Technology (2010).

[26] M. Byrne, M. A. Jimenez, J. C. Chavez, Predicting Well Inflow Using Computational Fluid Dynamics - Closer to the Truth?, Spe. 
[27] M. Byrne, M. A. Jimenez, E. A. Rojas, J. C. Chavez, G. D. F. Suez, Modelling Well Inflow Potential in Three Dimensions Using Computational Fluid Dynamics, Spe (128082).

[28] M. Byrne, M. A. Jimenez, E. A. Rojas, E. Castillo, Computational fluid dynamics for reservoir and well fluid flow performance modelling, SPE European Formation Damage Conference.

[29] F. A. Dumkwu, A. W. Islam, E. S. Carlson, Review of well models and assessment of their impacts on numerical reservoir simulation performance, Journal of Petroleum Science and Engineering 82-83 (2012) $174-186$.

[30] B. Goyeau, D. Lhuillier, D. Gobin, M. G. Velarde, Momentum transport at a fluid-porous interface, Int. J. Heat and Mass Transfer 46 (21) (2003) 4071-4081.

[31] F. Valdés-Parada, C. Aguilar-Madera, J. A. Ochoa-Tapia, B. Goyeau, Velocity and stress jump conditions between a porous medium and a fluid, Advan. Water Res. 62 (2013) 327-339.

[32] H. Li, S. A. Vasquez, M. Azhar, Modeling of multiphase flows in porous media with applications to reservoir and well performance analysis, Proceedings of the Asme Fluids Engineering Division Summer Meeting.

[33] P. Forchheimer, Wasserbewegung durch Boden, Zeitschrift des Vereines Deutscher Ingenieuer (45).

[34] R. Hooper, M. Hopkins, R. Pawlowski, B. Carnes, H. K. Moffat, Final Report on LDRD Project : Coupling Strategies for Multi-Physics Applications, Sand2007-7146.

[35] D. A. Lockerby, C. A. Duque-Daza, M. K. Borg, J. M. Reese, Time-step coupling for hybrid simulations of multiscale flows, J. Comp. Physics 237 (2013) 344-365.

[36] K.-A. Lie, S. Krogstad, I. Ligaarden, J. Natvig, H. Nilsen, B. Skaflestad, Open-source matlab implementation of consistent discretisations on complex grids, Comp. Geosciences 16 (2) (2012) 297322. 
[37] J. Olsen, C. S. Hemmingsen, L. Bergmann, K. K. Nielsen, S. L. Glimberg, J. H. Walther, Characterization and optimization of a nozzle-based inflow control device, SPE J. 32 (4) (2017) 224-233. 